\title{
Optical Design of Multimedia-Embedded LED Dental Astral Lighting using the Reverse Dual Reflector Method
}

\author{
Young Hoon Kwon, Hyo Chang Hwang, Hwa Joon Jun, and Jin Hyuk Kwon* \\ Department of Physics, Yeungnam University, Gyeongsan 712-749, Korea
}

(Received March 25, 2015 : revised June 3, 2015 : accepted June 3, 2015)

\begin{abstract}
Light-emitting diodes (LED) have many advantages for dental astral lighting because of their high color rendering index (CRI), low power consumption, light weight and longer life. A dental astral lamp is specially designed and simulated for securing the extra space for installing a multimedia display that will be helpful for treating young patients. The optical system using the reverse dual reflection method consisted of four illumination modules disposed at the four corners of the dental astral lamp, and each module comprises a high power LED, an elliptical mirror, and a multifacet reflector assembly using eight cylindrical mirrors. It is shown that the required illuminance, illumination pattern, and the illumination uniformity are well satisfied.
\end{abstract}

Keywords : Dental astral lamp, LED, High CRI, Optical system, Multimedia embedded

OCIS codes : (170.1850) Dentistry; (170.2945) Illumination design; (170.3890) Medical optics instrumentation; (120.4570) Optical design of instruments

\section{INTRODUCTION}

The dental astral lamp is a kind of special astral lamp used in medical treatment. It is mainly used to minimize the effect of shadow during surgery or diagnosis. High illuminance and large illumination area as well as the high color rendering index (CRI) are needed to minimize any errors in identifying the colors of the treatment area. Among the 15 color CRI values, in particular, the red color CRI (R9) is very important for the medical lamps in order to discriminate the color of blood. [1] The advantages of using LEDs as the light source of the dental astral lamp are the high CRI value, minimized heat, high color temperature, low power consumption, and longer life. The CRI value of the high power white LEDs has been increased due to development of phosphors that convert the blue LED lights into white light. [2, 3]

Besides the high CRI values, high illuminance is also required in the dental astral lamp. More specifically, the dental astral lamps require 10,000 $\mathrm{lx}$ for diagnosis and $2 \sim 40,000 \mathrm{~lx}$ for dental surgery. The appropriate illuminance for the dental astral lamp is about 20,000 lx. As shown in Fig. 1, the illumination pattern of the dental astral lamps is rectangular in contrast to the circular pattern of the general surgical astral lamps.

Most of the current dental astral lamps have been using halogen lamps as the light source, and so the strong heat as well as the large amount of stray light gave discomfort both to the dentist and the patient. Also, the color temperature and the CRI value are relatively low. The power consumption is large and the lifetime of the halogen lamp is very short compared to LEDs. [4] So, the dental astral lamp employing high power LEDs as the light source will be a good alternative for solving these problems.

The typical conventional astral lamp using the halogen lamp is based on the optical design of the elliptical mirror. The halogen lamp is located at the first focus and the target is located at the second focus of the elliptical mirror. [5] Besides there are other LED dental astral lamps that use a circular layout of several LED modules that employ many low power LEDs. [6] Many LED dental astral lamps use the same optical system as the halogen astral lamps except that the halogen lamp is replaced with the LEDs. [7]

In general the dental treatments are painful and generate fear in the patients because they are aware during treatment. Especially, small children show strong fear of dental treatment due to the sound and pain as well as having to look at the

\footnotetext{
*Corresponding author: jhkwon@ynu.ac.kr

Color versions of one or more of the figures in this paper are available online.
} 


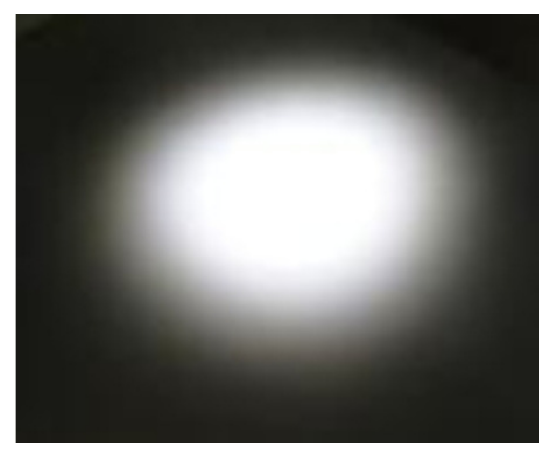

(a)

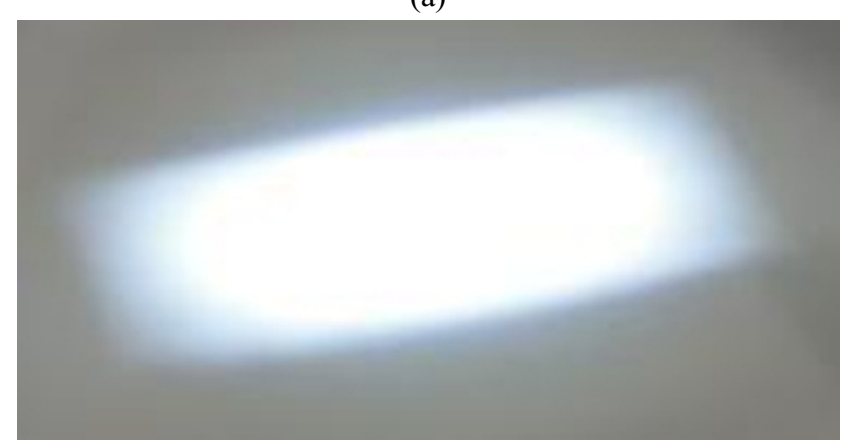

(b)

FIG. 1. Comparison of illumination patterns between (a) the general surgical astral lamp and (b) the dental astral lamp. Note that the surgical astral lamp generates a circular pattern compared to the rectangular pattern of the dental astral lamp.

dentist. Many dental clinics have begun to install multimedia facilities to decrease fear in children. Usually a display is installed at the ceiling and cartoons are shown there for the children to watch during dental treatment. However, installing a display on the high ceiling requires a large display and so it is expensive. Also it is very difficult to install displays that can be seen at the same time from several dental chairs.

It was very difficult to design a dental astral lamp with enough space to install a multimedia display on the dental astral lamp when the halogen lamp was used as the light source because the halogen lamp is large. However, the small size and high brightness of high power LEDs make a special optical design possible due to the degree of freedom in the structural design. [8] Since the light source module can be made very small by using the LEDs, it is possible to secure enough space for the multimedia by adopting the reverse dual reflection method in which the rays are emitted in the opposite upward direction compared to the downward emission of the conventional astral lamp.

In this study we report an optical design of a new dental astral lamp equipped with a multimedia display at the center of the dental astral lamp by utilizing the extra space secured by using the high power LED modules and the reverse dual reflection method. This new design will give the dentist more space to treat the patient as well as providing comfort to the patient during treatment.

\section{OPTICAL DESIGN AND SIMULATION}

The important optical specifications of the dental astral lamp are the illumination pattern size, the illuminance uniformity, the color temperature, and the CRI value. The typical distance between the dental astral lamp and the patient is given as $700 \mathrm{~mm}$. [10] The color temperature and the CRI value are determined by the LED itself, and the total illuminance is determined by the number of LEDs and LED brightness. The illumination pattern and uniformity are determined by the optical system design. The illuminance uniformity is defined by

$$
D_{50} / D_{10}=\frac{\text { Diameter at } 50 \% \text { intensity of peak illuminance }}{\text { Diameter at } 10 \% \text { intensity of peak illuminance }} \times 100(\%)
$$

The typical illumination profile has the Gaussian shape with top hat profile, and generally it is required that $D_{50} /$ $D_{10}$ value should have about $60 \sim 70 \%$ to ensure comfort to the patient by minimizing the stray light during treatment. The size of the illumination pattern on the target is determined by the $D_{10}$ value and the light falling outside of $D_{10}$ is regarded as the lost light. To prevent the lost light falling on the eyes of the patient, which can generate discomfort, they should be minimized.

A 7-inch display is assumed to be placed at the center of the dental astral lamp as shown in Fig. 2. This configuration would be impossible with the conventional halogen astral lamp but it is possible to secure the extra space at the center by employing the small LED sources and the reverse dual reflection method together. The reverse dual reflecting structure as shown in Fig. 2 is accomplished by placing the LEDs at the rear of the multimedia display, and the beam characteristics are determined by dual reflections by the elliptical mirror and the multifacet reflector assembly. Firstly the elliptical reflector expands and determines the illumination spot size and then the multifacet reflector assembly that is composed of eight cylindrical reflectors forms the asymmetrical beam pattern.

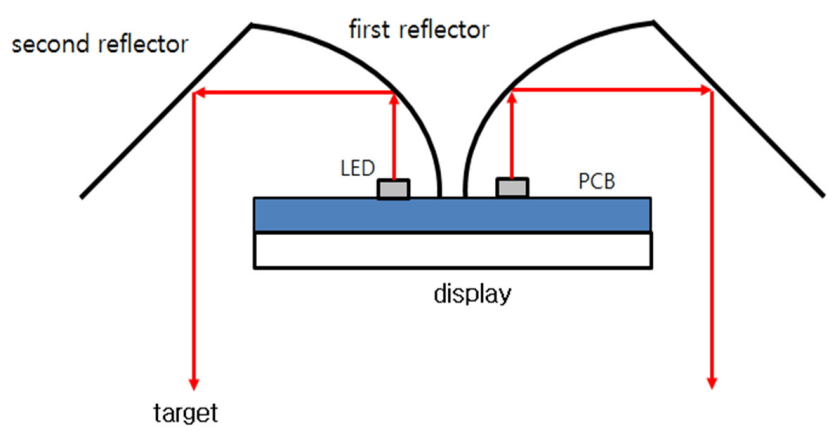

FIG. 2. Schematic of the beam paths of the optical modules in the dental astral lamp that is equipped with a display at the center. 
The target specification of the optical design is given as follows,

1. Illuminance : $>25,000 \mathrm{~lx}(4 \mathrm{~W}$ LED)

2. Illumination area : $150 \mathrm{~mm} \times 90 \mathrm{~mm}$ (rectangular)

3. Illumination uniformity : D50 / D10 > 65\%

The KS standard requires the central illuminance to be between 8,000 20,000 lx but most commercial dental astral lamps provide the maximum illuminance larger than 25,000 lx with the function of controlling the illuminance. Another requirement of the KS standard is that the central area with horizontal diameter $50 \mathrm{~mm}$ should be larger than $75 \%$ of the maximum illuminance. [11]

The optical simulation was performed using the SPEOS CATIA optical design program that incorporated the optical design program SPEOS and the computer-aided design (CAD) program CATIA into a single code. The optical specification of the LED was obtained from the rayfile data provided by Osram and the reflectance of the reflectors was assumed to be $90 \%$.

The LEDs are four Osram's LCW-CRDP LED (1W) that have a high CRI value of 96. [8] To produce the rectangular field size of about $150 \mathrm{~mm} \times 90 \mathrm{~mm}$ at a distance of $700 \mathrm{~mm}$ from the LED light source the elliptical mirror was used as the first reflecting surface. The LED source was assumed to be located near the first focal point of the elliptical mirror with a small focus offset, and the target was assumed to be located at the second focal point of the elliptical mirror. The beam from the LED that was emitted toward the reverse direction was deflected 90 degrees by the elliptical surface. The specifications of elliptical reflector as shown in Fig. 3 are as follows: the distance from the edge of the elliptical reflector to the focal point is $7.5 \mathrm{~mm}$, the distance from first focus to second focus $700 \mathrm{~mm}$, and the opening height $33.6 \mathrm{~mm}$.

The focus offset position of the LED source as shown in Fig. 3 was changed from $-1.0 \mathrm{~mm}$ to $+1.0 \mathrm{~mm}$ in the interval of $0.2 \mathrm{~mm}$ and the maximum illuminance and the illuminance uniformity were obtained as shown in Figs. 4 and 5. As shown in Fig. 4, the focus offset in the negative

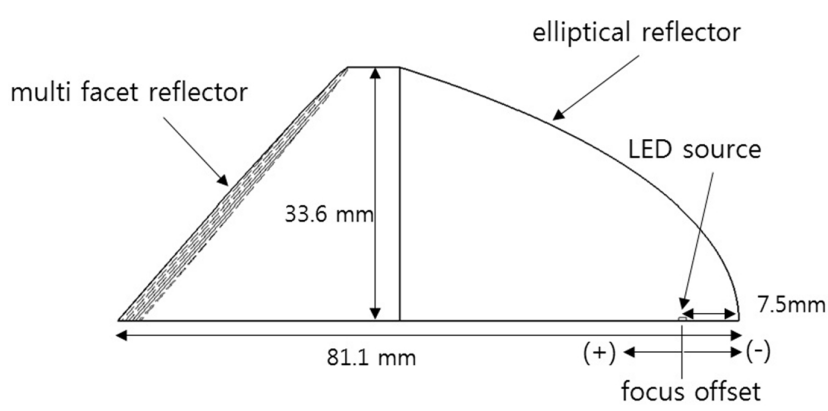

FIG. 3. The optical design of the elliptical reflector. The LED source is located $7.5 \mathrm{~mm}$ from the edge of the reflector, and the aperture width is $33.6 \mathrm{~mm}$. range gave very poor illuminance uniformity and the best focus offset satisfying both the maximum illuminance, the vertical pattern size, and the illuminance uniformity was $+0.6 \mathrm{~mm}$. When the focus offset position was larger than $0.6 \mathrm{~mm}$, the illuminance uniformity was deteriorated severely as shown in Fig. 5.

The rectangular beam pattern was accomplished by dividing the second reflecting surface into multifacet cylindrical reflectors that have curvatures along one direction as shown in Fig. 6. The multifacet reflector assembly consisted of 8 reflecting cylindrical reflectors; each cylindrical reflector had the same width of $10 \mathrm{~mm}$ and different radii of curvature to achieve the rectangular beam pattern at the target by adjusting the spread angle from 0 to 3 degrees. Figure 7 shows the change of the rectangular beam pattern in terms of the spread angle. All the cylindrical reflectors were tilted 3.3 degrees from the normal direction toward outside to direct the LED light from the elliptical reflector toward the target. The tilt angle was obtained by dividing the half of the separation $80 \mathrm{~mm}$ between two LED illumination

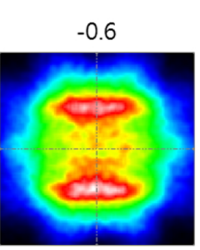

0.2

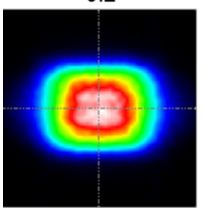

$-0.4$

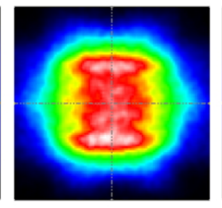

0.4

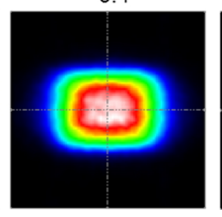

unit : $\mathrm{mm}$

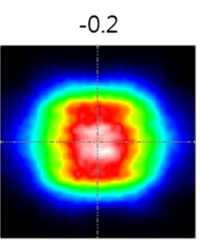

0.6

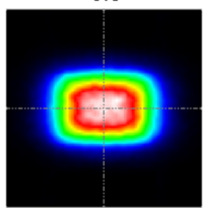

0

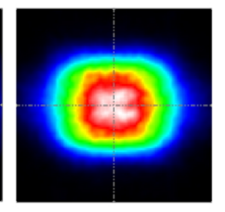

0.8

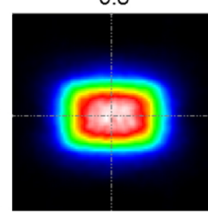

FIG. 4. The change of illuminance pattern as the focus offset of the LED source is changed from $-0.6 \mathrm{~mm}$ to $+0.8 \mathrm{~mm}$.

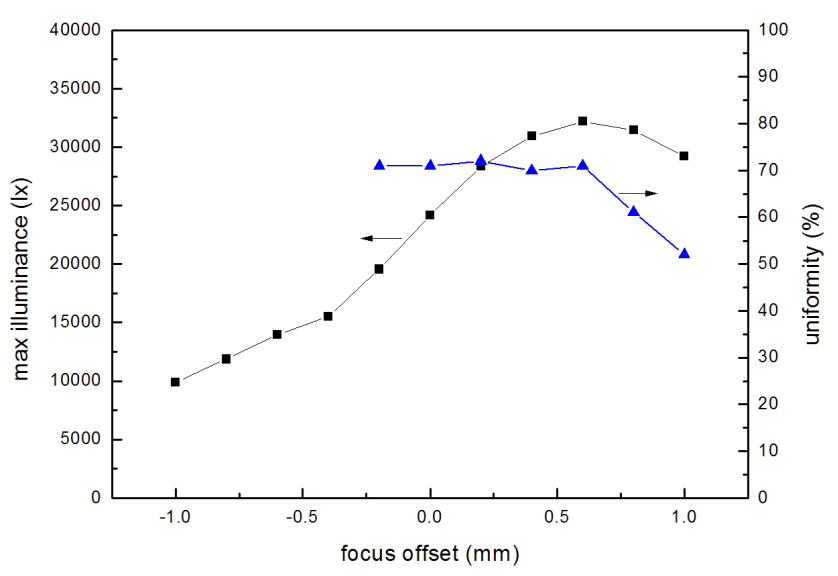

FIG. 5. The change of the maximum illuminance and the illuminance uniformity in terms of the focus offset. The optimal focus offset is $+0.6 \mathrm{~mm}$. 


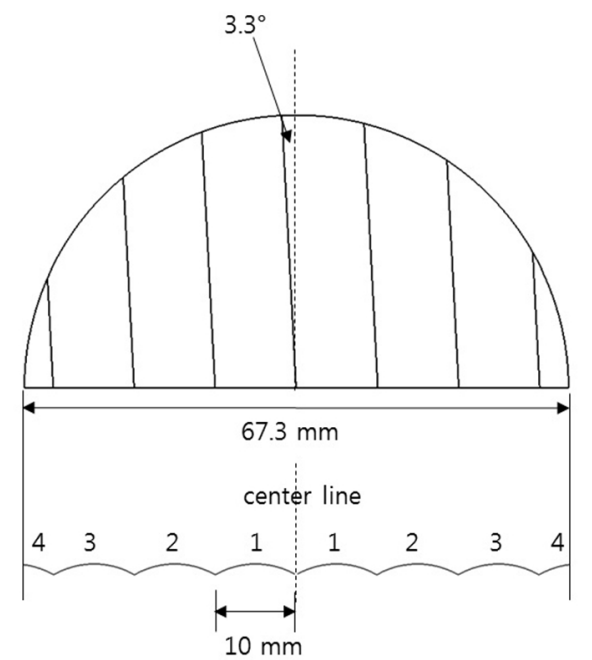

FIG. 6. The optical design of the multifacet cylindrical reflector unit consisting of 8 cylindrical reflectors. The radii of curvature of the cylindrical reflectors are $R_{1}=59.3 \mathrm{~mm}, R_{2}=$ $62.2 \mathrm{~mm}, R_{3}=68.1 \mathrm{~mm}$, and $R_{4}=77 \mathrm{~mm}$ from the center to outside, respectively.

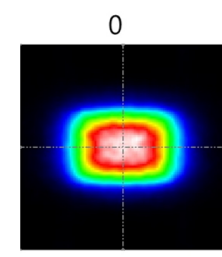

2.0
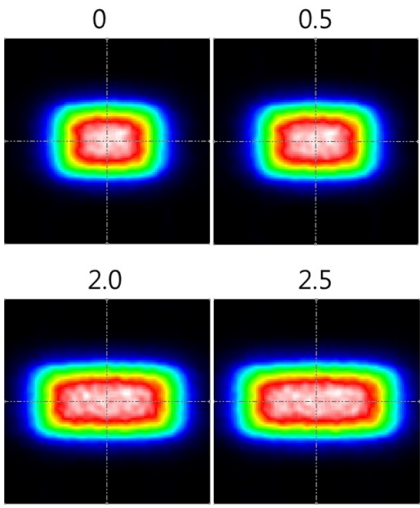

2.5
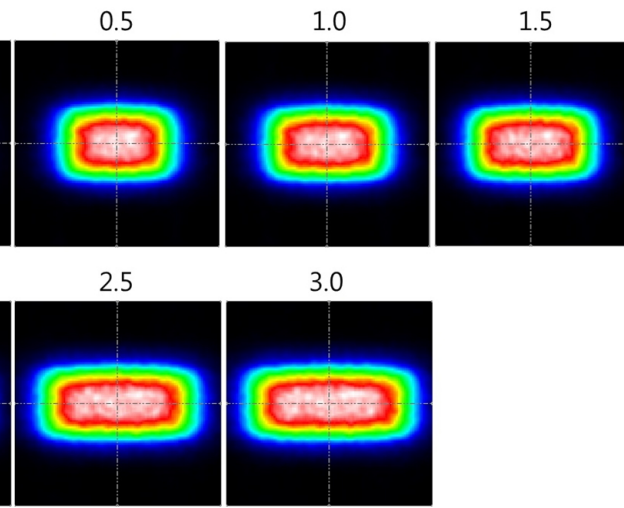

3.0

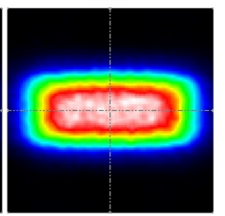

FIG. 7. The change of the illuminance pattern as the spread angle is changed from zero to 3.0 degrees. The optimum spread angle satisfying the target design of $150 \mathrm{~mm} \times 90 \mathrm{~mm}$ is 1 degree. modules with the distance $700 \mathrm{~mm}$ to the target. Table 1 shows the radii of curvature, the maximum illuminance, the horizontal and vertical $D_{10}$ values, and the horizontal and vertical uniformities $D_{50} / D_{10}$, respectively. The best spread angle satisfying the target specification was obtained with the spread angle of 1 degree, and the radii of curvature were $R_{l}=59.2 \mathrm{~mm}, R_{2}=62.2 \mathrm{~mm}, R_{3}=68.1 \mathrm{~mm}, R_{4}=77$ $\mathrm{mm}$, and the vertical and horizontal illuminance uniformities were $71 \%$.

The exit aperture size that is determined by the size of the multifacet reflector assembly is about $67.3 \mathrm{~mm} \times 33.6$ $\mathrm{mm}$ in the semicircle shape. Also, the multifacet reflecting assembly was tilted 45 degrees in order to reflect the incoming beam from the first elliptical reflector toward the target.

Figure 8 (a) shows the ray tracing diagram of the single illuminating module that is comprised of the $1 \mathrm{~W}$ LED, the elliptical reflector, and the multifacet reflector assembly. Figure 8 (b) shows the exterior shape the single illumination module. The LED dental astral lamp consists of 4 illuminating modules that are disposed at the four corners of the dental astral lamp as shown in Fig. 9.

\section{RESULTS AND DISCUSSION}

Figure 10 shows the illumination profiles of the beams at the target. The central illuminance at the target is $26,220 \mathrm{~lx}$, that is about $4.9 \%$ higher than the target value of 25,000 lx. The total flux at the target is $183 \mathrm{~lm}$ that corresponds to $53 \%$ power efficiency from the LEDs to the target. The illumination uniformity $D_{50} / D_{10}$ was $71 \%$ along the horizontal and the vertical direction both satisfying the target value of $65 \%$. The illumination area $D_{10}$ at the target is $153 \mathrm{~mm}$ $\times 90 \mathrm{~mm}$ along the horizontal and vertical directions, which matches the adequate rectangular shape needed for the dental treatment.

The dental astral lamp comprises the four LED illumination modules disposed at the reverse side of the dental astral lamp and can supply most of its light onto the mouth area,

TABLE 1 . The radii of curvature of the multifacet cylindrical reflectors, the maximum illuminance, and the illuminance uniformities obtained from the optical simulation in terms of the spread angle. The spread angle of 1 degree gave the best optimization satisfying the design target

\begin{tabular}{c|c|c|c|c|c|c|c|c|c|c}
\hline \hline Spread angle & $R_{1}$ & $R_{2}$ & $R_{3}$ & $R_{4}$ & Maxillum. & $D_{10}(H)$ & $D_{10}(V)$ & $D_{50} / D_{10}(H)$ & $D_{50} / D_{10}(V)$ \\
\hline deg & \multicolumn{5}{|c|}{$\mathrm{mm}$} & \multicolumn{3}{|c|}{$\mathrm{mm}$} & \multicolumn{2}{c}{$\%$} \\
\hline 0 & 71.6 & 75.6 & 83.6 & 96.2 & 32178 & 135 & 90 & 67 & 71 \\
\hline 0.5 & 64.9 & 68.3 & 75.1 & 85.6 & 29343 & 144 & 90 & 69 & 71 \\
\hline $\mathbf{1}$ & $\mathbf{5 9 . 3}$ & $\mathbf{6 2 . 2}$ & $\mathbf{6 8 . 1}$ & $\mathbf{7 7}$ & $\mathbf{2 7 0 2 9}$ & $\mathbf{1 5 3}$ & $\mathbf{9 0}$ & $\mathbf{7 1}$ & $\mathbf{7 1}$ \\
\hline 1.5 & 54.6 & 57.2 & 62.3 & 70.1 & 25052 & 162 & 91 & 73 & 70 \\
\hline 2 & 50.6 & 52.9 & 57.5 & 64.3 & 23270 & 172 & 91 & 74 & 70 \\
\hline 2.5 & 47.2 & 49.3 & 53.3 & 59.4 & 21612 & 181 & 91 & 76 & 70 \\
\hline 3 & 44.2 & 46.1 & 49.7 & 55.1 & 20141 & 191 & 91 & 78 & 70 \\
\hline
\end{tabular}




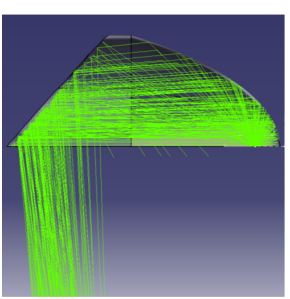

(a)

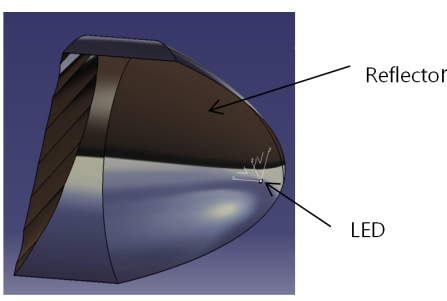

(b)
FIG. 8. (a) The ray tracing of the illuminating module using the SPEOS code and (b) the exterior shape designed by the CATIA CAD code.

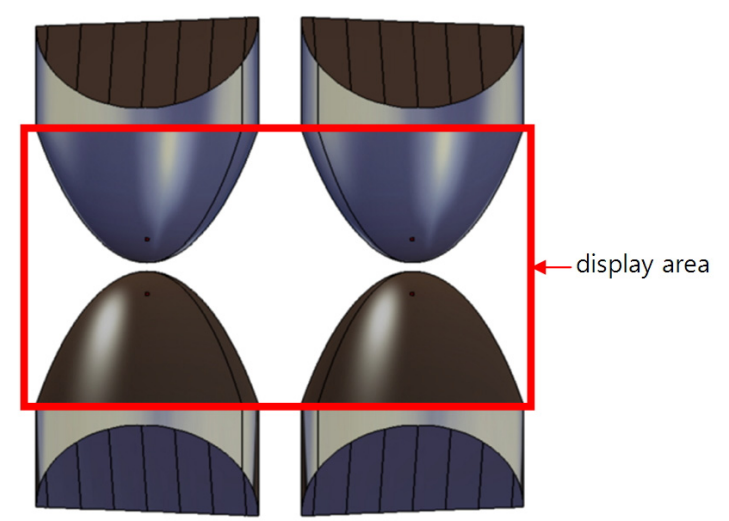

FIG. 9. The top view of the dental astral lamp that is equipped with four LED illumination modules at the four corners and a seven-inch display at the central area.

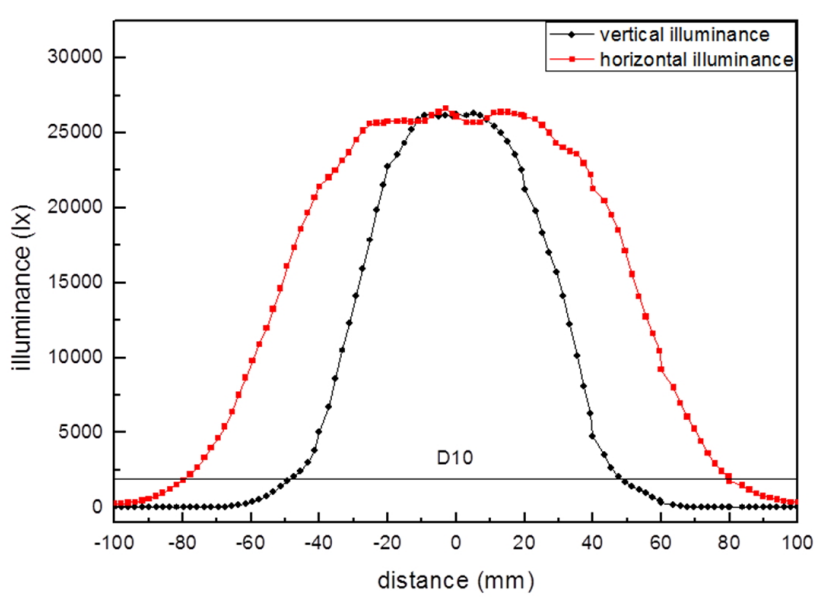

FIG. 10. The vertical and horizontal illuminance distribution at the target. D10 line defines the size of the illumination to be $150 \mathrm{~mm}(\mathrm{H}) \times 90 \mathrm{~cm}(\mathrm{~V})$.

making it possible to secure an extra space for the 7-inch display at the center of the dental astral lamp.

The glare of the LED dental astral lamp comprising 4 illumination modules at the four corners is estimated to be much less than the conventional halogen astral lamp or the direct illuminating LED lamps because the lights are dispersed

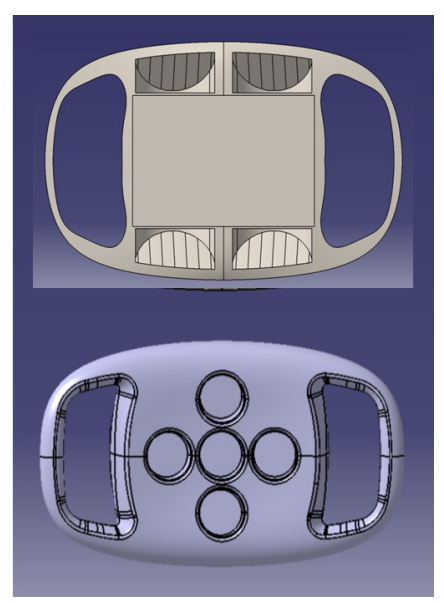

FIG. 11. Comparison of the multimedia embedded LED dental astral lamp (up) with the conventional LED astral lamp (down). Note that the conventional LED astral lamp does not have any space for the multimedia display.

at the four corners with lower peak intensity. The estimated illuminance at the eye was about $10 \mathrm{~lx}$ from the simulation and this is low enough to provide comfort to the patient compared to the strong glare of the halogen astral lamp that is generating stray light due to the high intensity of the halogen lamp and scattering at the mirror surface.

This will enable child patients to watch a movie or cartoon to minimize the fear of dental treatment. Also, the optical characteristics of the LED dental astral lamp are better than the conventional halogen astral lamp because LEDs with high luminosity and CRI were used as the light sources. The discomfort induced by the haze of the dental astral lamp is also minimized because the high brightness LEDs are hidden in the backside of the dental astral lamp and only the highly specular multifacet reflector assemblies with a large aperture are exposed at the four corners. The thermal discomfort is also minimized because the direct thermal radiation of the LED is much less than that of the halogen lamps. In the aspect of industrial manufacturing the LED dental astral lamp is lighter and smaller in weight and volume besides the advantage of extra space for the 7 inch display as compared with the conventional LED dental astral lamp that has no space for the multimedia display as shown in Fig. 11.

\section{CONCLUSION}

An LED dental astral lamp that is equipped with a 7-inch display is designed by using the reverse dual reflector method. Four illumination modules comprising the LED, the elliptical reflector, and the multifacet reflector assembly are disposed at the four corners of the LED dental astral lamp. The optimal focus offset position of the LED source and the spread angle of the multifacet reflector assembly are shown to be $+0.6 \mathrm{~mm}$ and 1 degree. Optical characteristics 
as well as the mechanical design such as the lamp size and the weight and the thermal discomfort are shown to be much better than for the conventional halogen or LED dental astral lamps. The requirement of the KS standard for the glare is the illuminance at the distance of the 60 $\mathrm{mm}$ should be less than 1,200 $\mathrm{lx}$ and our simulation showed that the illuminance at this distance is only $435 \mathrm{~lx}$, which is much less than the requirement. Besides the simulation showed that the illuminance at the eye is estimated to be as low as $10 \mathrm{~lx}$, which is possible by using the LED and the reverse dual reflector method compared to the simple elliptical reflector that is used in the conventional halogen astral lamp.

\section{ACKNOWLEDGMENT}

This work was supported by the 2013 Yeungnam University Research Grant.

\section{REFERENCES}

1. N. Kimura, K. Sakuma, S. Hirafune, K. Asano, N. Hirosaki, and R. Xie, "Extra high color rendering white light-emitting diode lamps using oxynitride and nitride phosphors excited by blue light-emitting diode," Appl. Phys. Lett. 90, 051109 (2007).
2. H.-Y. Ryu and D.-H. Kim, "High-brightness phosphorconversion white light source using InGaN blue laser diode," J. Opt. Soc. Korea 14, 415-419 (2010).

3. H.-Y. Ryu, "Analysis on the luminous efficiency of phosphorconversion white light-emitting diode," J. Opt. Soc. Korea 17, 22-26 (2013).

4. R. Guan, D. Tian, and X. Wang, "Design and implementation of LED daylight lamp lighting system," in Proc. ICEPT-HDP (Riverfront Business Hotel, China, July 2008), pp. $1-3$.

5. J. H. Mun, H. J. Lee, and G. T. Hong, "Optimum design of an astral lamp for dental surgery using high power LED," in Proc. KSPE (Ramada Plaza Jeju Hotel, Korea, June 2009), pp. 403-404.

6. W. Y. Cheon, S. B. Song, J. H. Kim, J, H, Kim, and J. W. Park, "Development of LED operating light," in Proc. KIEE (Muju Resort, Korea, July 2009), pp. 1617-1618.

7. Y. H. Kwon, "Technical trend of LED dental lighting," KIEEME Digest Tech. Papers 25, 20-24 (2012).

8. D. A. Steigerwald, J. C. Bhat, D. Collins, R. M. Fletcher, M. O. Holcomb, M. J. Ludowise, P. S. Martin, and S. L. Rudaz, "Illumination with solid state lighting technology," IEEE J. Select. Topics Quantum Electron. 8, 310-320 (2002).

9. C. C. Hsieh and Y. H. Li, "The development of LED-based dental light using a multiplanar reflector design," International Journal of Photoenergy 2014, 9575079 (2014).

10. http://www.osram-os.com/osram_os/en/products/product-catal og/leds-for-general-lighting/oslon-ssl-150-white/lcw-crdp.cc/i ndex.jsp

11. KS Standard 5.2.2.2. 